\title{
JUBILACIÓN: CAMBIOS PERCIBIDOS EN RELACIÓN A RUTINAS Y ROLES EN ADULTOS MAYORES NO INSTITUCIONALIZADOS DE LA CIUDAD DE PUNTA ARENAS.
}

\author{
RETIREMENT: NOTICED CHANGES IN RELATION TO ROUTINE AND \\ ROLES IN ELDER PEOPLE NON- INSTITUTIONALIZED FROM PUNTA \\ ARENAS CITY.
}

\author{
Igor Alvarado, Andrea Paola ${ }^{1}$ Lara Huerta, Carola Andrea ${ }^{2}$ Ortega Díaz, Verónica Andrea $^{3}$ \\ Vallejos González, Karla Waleska ${ }^{4}$
}

\begin{abstract}
RESUMEN
Esta investigación tiene como principal propósito identificar las percepciones de adultos mayores no institucionalizados de la ciudad de Punta Arenas, respecto a los cambios producidos en sus rutinas y roles luego del proceso de jubilación, desde el enfoque de la Ciencia de la Ocupación y la Terapia Ocupacional en la región de Magallanes y Antártica Chilena.

La metodología empleada tiene un enfoque cualitativo. Para la recolección de datos, se utilizó una entrevista semiestructurada y el análisis de información se basa en la Teoría Fundamentada de Glaser \& Strauss (1967).

Como principales resultados, se obtiene que la existencia de cambios en las rutinas y roles, incluyen percepciones positivas y negativas asociadas. Además, se identifica que en el desempeño de rutinas y roles de las personas, existe una estrecha relación.

Finalmente, se puede concluir que para los adultos mayores, la adaptación a dichos cambios producidos después de vivir el proceso de jubilación, depende de las oportunidades de involucramiento en actividades significativas, junto con la posibilidad de contar con redes de apoyo.
\end{abstract}

Palabras Claves: Adulto mayor, jubilación, cambios percibidos, roles y rutinas.

\begin{abstract}
This research has as main purpose to identify the perception of non institutionalized elderly people from Punta Arenas city, on the matter of all changes produce in their routines and roles after the retirement process; seeing from the approach of Occupation's Science and Occupational Therapy in the Magellan and Antarctic Chilean region.

The methodology used has a qualitative approach. It was used a semi-structured interview for the data collection, and the analysis information is based upon The Grounded Theory of Glaser \& Strauss (1967).

As main results, it was obtained that the existence of changes in the routines and roles, include positive and negative perception associated. Furthermore, it was identified that a narrow relationship exists in the performance of the routines and roles of the people.

Lastly, it can be concluded that for elderly people, the adaptation to those changes produced after living the retirement process depends on the opportunities of getting involved in meaningful activities, together with the possibility of counting with support networks.
\end{abstract}

Key words: Older people, retirement, changes perceived, roles and routines.

\footnotetext{
${ }^{1}$ Licenciada en Ciencia de la Ocupación, Terapia Ocupacional, Universidad de Magallanes. igor.andrea@hotmail.cl

${ }^{2}$ Licenciada en Ciencia de la Ocupación, Terapia Ocupacional, Universidad de Magallanes. carola.lara.huerta@gmail.com

${ }^{3}$ Licenciada en Ciencia de la Ocupación, Terapia Ocupacional, Universidad de Magallanes. verito.ortega@ hotmail.com

${ }^{4}$ Licenciada en Ciencia de la Ocupación, Terapia Ocupacional, Universidad de Magallanes. vallejos.karla@gmail.com
} 


\section{INTRODUCCIÓN}

El presente estudio se focaliza en un grupo de adultos mayores de la ciudad de Punta Arenas, Chile.

Este grupo etario, a nivel mundial, ha experimentado un crecimiento exponencial en respuesta a numerosos factores que se han conjugado para favorecer una mayor esperanza de vida. Según el Instituto Nacional de Estadísticas, en el último CENSO realizado en el año 2002, se señaló que la población correspondiente a personas mayores de 60 años de edad correspondía a 17.026 habitantes en la XII Región de Magallanes y la Antártica Chilena (Moreno, E. \& Miles, J. 2003).

En este sentido, es importante considerar el concepto de envejecimiento poblacional, proceso que comienza a manifestarse tanto a nivel regional, nacional y mundial. Debido a esto, es importante que los profesionales de la salud y la población en general tengan conocimiento de los efectos del proceso de envejecimiento en las personas, su percepción de bienestar y calidad de vida, teniendo en cuenta que el envejecimiento poblacional seguirá incrementándose en forma sistemática.

Por su parte, según el Marco para la Práctica de la Terapia Ocupacional (AOTA, 2010), los patrones de desempeño se refieren a los hábitos, rutinas, roles y rituales utilizados en el proceso de participar en las ocupaciones o actividades (AOTA, 2010). En particular, las rutinas se definen como secuencias establecidas de ocupaciones o actividades que proveen de estructura de la vida diaria, las cuales también pueden promover o afectar la salud (Fiese et al., 2002; Segal, 2004), y los roles como un conjunto de comportamientos que tiene una función social acordada y para los cuales existen códigos normativos aceptados (Christiansen y Baum, 1997).

Ahora bien, normalmente las personas deben alcanzar una edad legal para que se produzca el cese laboral, lo que genera un cambio en la rutina y los roles que desempeñan. Además, dejan de recibir la retribución por el trabajo realizado por el resto de su vida, disminuyendo considerablemente el ingreso económico de esa persona, a pesar de obtener algún tipo de pensión. Esta nueva condición puede incidir en una variación importante en los patrones de desempeño de las personas, pudiendo afectar directamente en sus rutinas, hábitos, roles y su participación en rituales.

Teniendo estos antecedentes es trascendental identificar cómo los sujetos perciben este cambio después de haber jubilado, considerando que la ciudad de Punta Arenas posee ciertas particularidades (geográficas, climáticas, entre otras), las que pudiesen influir en los patrones de desempeño de las personas, teniendo en cuenta además que no se han realizado estudios o investigaciones al respecto.

A partir de lo anterior, se plantea como objetivo que guía esta investigación el conocer la percepción de los adultos mayores con respeto a los cambios producidos en sus patrones de ejecución posterior a la jubilación, en personas no institucionalizadas residentes de la ciudad de Punta Arenas. A partir de éste, se desprenden los siguientes objetivos específicos: Describir los significados asociados a los cambios producidos en las rutinas de los adultos 
mayores jubilados y describir los significados asociados a los cambios producidos en los roles de los adultos mayores jubilados.

Este estudio fue abordado desde la perspectiva de la Ciencia de la Ocupación, utilizando conceptos desde la visión de la Terapia Ocupacional, estableciendo la importancia de la ocupación como medio y fin del bienestar; teniendo en cuenta la naturaleza de la ocupación y su relación con la salud (Crepeau, Cohn, Schell, 2011.).

\section{METODO}

En este estudio se utilizó un enfoque cualitativo, el cual según Taylor y Bogdan (1996), se refiere en su más amplio sentido a "la investigación que produce datos descriptivos: Las propias palabras de las personas, habladas o escritas, y la conducta observable" (Taylor, S. J., \& Bogdan, R. 1987). Además, este enfoque permite enfatizar y proveer un entendimiento comprensivo del entorno social y contextual que ayudará a comprender de mejor manera el fenómeno en cuestión.

El diseño elegido es exploratorio-descriptivo: Es exploratorio, porque el conocimiento sobre el tema es escaso en la Región de Magallanes y Antártica Chilena, por lo tanto, este tipo de diseño permite descubrir las bases y recabar información que dé como resultado del estudio la formulación de una hipótesis. Es también descriptiva, porque permite conceptualizar y caracterizar los diversos aspectos del fenómeno de estudio, con el fin de establecer su estructura o comportamiento (Taylor, 1987)

Los criterios considerados para definir la población de estudio de esta investigación fueron: Ser adulto mayor (tanto hombres como mujeres, mayores de 60 años); encontrarse al menos un año retirado laboralmente (jubilado); no encontrarse institucionalizado; tener como residencia actual la ciudad de Punta Arenas; aceptar voluntariamente ser parte de la investigación; no poseer trastornos de salud mental, como demencias o Alzheimer, ya que pueden interferir en los resultados. La caracterización general de los actores consultados se expone en la Tabla $\mathrm{N}^{0} 1$. 
Tabla $\mathrm{N}^{\circ}$ 1: Caracterización de los participantes en estudio.

\begin{tabular}{|l|l|l|c|c|c|}
\hline Participante & Edad & Género & $\begin{array}{c}\text { Años de } \\
\text { jubilación }\end{array}$ & $\begin{array}{c}\text { Tipo de } \\
\text { jubilación }\end{array}$ & $\begin{array}{c}\text { Trabajo que } \\
\text { realizaba }\end{array}$ \\
\hline Entrevistado 1 & 63 años & Hombre & 1 año & Anticipada & $\begin{array}{c}\text { Jefe de Cuadrilla } \\
\text { (ENAP) }\end{array}$ \\
\hline Entrevistado 2 & 69 años & Hombre & 4 años & Normal & $\begin{array}{c}\text { Operario de } \\
\text { máquinas } \\
\text { (FAMAPAL) }\end{array}$ \\
\hline Entrevistado 3 & 63 años & Hombre & 1 año & Normal & $\begin{array}{c}\text { Operario de } \\
\text { máquinas (ENAP) }\end{array}$ \\
\hline Entrevistado 4 & 66 años & Hombre & 8 años & Normal & $\begin{array}{c}\text { Empleado Fuerza } \\
\text { Aérea }\end{array}$ \\
\hline Entrevistado 5 & 67 años & Mujer & 5 años & Anticipada & $\begin{array}{c}\text { Auxiliar de } \\
\text { Enfermeria }\end{array}$ \\
\hline Entrevistado 6 & 62 años & Mujer & 1 año & Anticipada & $\begin{array}{c}\text { Corredora de } \\
\text { Propiedades }\end{array}$ \\
\hline
\end{tabular}

Fuente: Autoras

Como muestra la Tabla $\mathrm{N}^{\circ} 1$, la investigación contempló a 6 adultos mayores, hombres y mujeres, con una edad promedio de 65 años y con un promedio de 3 años de jubilación. Se incluyen también los tipos de jubilación a la que acceden los entrevistados (normal y anticipada), las cuales hacen referencia a jubilar luego de la edad establecida, o bien, en forma anticipada debido a diferentes causas médicas. Por último, se muestra el trabajo que realizaban los entrevistados antes del proceso de jubilación, ya que muchos estaban sometidos a jornadas laborales extensas, donde su rutina diaria se basaba en cumplir turnos que los obligaban a permanecer fuera de su hogar. Dicho elemento es relevante para esta investigación, debido a que se producen significativos cambios en las percepciones que poseen las personas luego de la jubilación.

Como instrumento de recolección de datos, en este estudio se utilizó la entrevista de tipo semiestructurada, donde los principales focos de atención se centraron en las rutinas y roles de las personas mayores participantes. La construcción del instrumento utilizado se realiza de acuerdo a los objetivos presentados con anterioridad, buscando obtener información clave respecto a temáticas centrales como los significados asociados a los cambios producidos en las rutinas y roles. Por los antes mencionado, se levantan 2 ejes temáticos los cuales son: Cambios asociados a las rutinas y cambios asociados a los roles.

El método de análisis de datos que se utilizó en esta investigación fue The Grouded Theory de Glaser y Strauss en 1967, la cual consiste en "descubrir la teoría desde los datos a través de un método general de análisis comparativo" (Glaser, B. \& Strauss, A. 1967).

En el análisis de la presente investigación, se utilizaron las 3 etapas de codificación de la información planteadas por esta teoría: Codificación abierta, codificación axial y codificación selectiva. 
Además, es importante mencionar las consideraciones éticas de esta investigación, las que contemplaron las siguientes: los participantes tenían pleno conocimiento de sus derechos mediante la lectura, aprobación y firma de un consentimiento informado, el que explicitaba su derecho a la confidencialidad, derecho a la reserva de información o de no continuar con la entrevista, derecho a conocer los resultados de la investigación realizada y la autorización de publicar los resultados de la misma.

\section{RESULTADOS}

\section{Eje Temático I: Cambios asociados a las rutinas}

Como resultado del análisis de este primer eje temático, se obtiene que los entrevistados perciben que su etapa de vida laboral activa no les permitía la organización de sus tiempos, puesto que dependían en gran medida de factores externos. Posteriormente, al jubilarse, cada persona organiza en forma individual sus tiempos, reorganizando su rutina habitual, acarreando como consecuencia percepciones diferentes, positivas o negativas respecto al proceso vivido.

\section{Percepciones positivas:}

- Satisfacción personal:

"...ah, ahora lo estoy haciendo feliz, feliz, porque ya cumplí con lo que tenía que cumplir, nada más, eso" (E1,H,63,L121).

- Tranquilidad:

"Ahora estoy tranquilo, si la única que cambia es la rutina(...), yo me despedía de mis hijos, pero no sabía si iba a volver, ahora no poh, ahora uno está tranquilo en la casa y es bonito poh (suspira), es precioso estar con toda su gente" (E1,H,63,1103).

- Participación social:

"Hago mi repostería que me encanta...entonces entrego algunos dulces para afuera...vendo joyas, así que soy bien como activa igual, dentro de. Si, no sirvo para estar sin hacer nada, si, ¡todavía no!” (E6,M,62,L60).

- Adaptación:

“...uno se estructura y después como que te acostumbras, ponte tú...tu reloj biológico sigue funcionando pero me quedo en cama, veo tele, ya no estoy mirando la hora para irme...sobre todo en invierno, que rico estar en la cama, no tener que estar saliendo" (E6,M,62,L67).

La mayor parte de los actores consultados identifican que tanto su participación social, su adaptación al proceso de jubilación y la tranquilidad que logran percibir con este cambio, les producen satisfacción personal. Por otra parte, al realizar actividades de participación social, refiriéndose a actividades sociales a aquellas actividades en que interactúan con otros y establecen lazos, posterior a la jubilación, con respecto al tiempo libre del cual 
disponen. De acuerdo a esto las personas logran una mejor adaptación al proceso de cambio; y a partir de esta adaptación se genera un sentimiento de tranquilidad. Las percepciones positivas que surgen en los individuos se deben a la percepción de contar con más tiempo para organizar sus rutinas y realizar actividades significativas cotidianamente.

\section{Percepciones negativas:}

- Dificultad:

"me costó superarlo los primeros tres meses, pero después ya de eso ya la rutina del día a día prácticamente es estar con mi señora, con mis hijos y mis nietos que me visitan" (E3,H,63,L80).

- Preocupación:

"Pasar a ser un pensionado, un jubilado, sin ninguna carga de trabajo laboralmente hablando y eso me llevó a que haya estado unos dos o tres meses con la idea de que... ¿cómo lo voy a hacer?, ¿qué es lo que tengo qué hacer?” (E3,H,63,L71).

Las principales percepciones negativas expresadas por los entrevistados tienen relación con el proceso de cambio que tuvieron durante los primeros meses post jubilación, evidenciado como desconcierto y sensación de no saber en qué ocuparse y cómo organizar su tiempo libre. Estos elementos influyen entre sí en el desempeño de la nueva rutina diaria del adulto mayor jubilado.

\section{Eje Temático II: Cambios asociados a los roles}

En relación a los cambios asociados a los roles, las personas después de jubilar presentan cambios en este ámbito, en donde habrían roles que ya no se desempeñan y otros que se potencian, así como también, una modificación en el tiempo dedicado a cada uno de ellos. A través del análisis se demuestra de manera positiva y negativa la percepción que tiene cada entrevistado.

\section{Percepción positiva:}

- Satisfacción antes de jubilar:

"Fui dirigente sindical de terreno por muchos años, eso también me ayudó mucho a sobrepasar o a estar en una situación bastante privilegiada entre comillas" (E3,H,63,L52). 
- Satisfacción después de jubilar:

"Ahora estoy disfrutando mucho más el rol de mamá por supuesto, de abuela..." (E6,M,62,L74).

Los sujetos creen cumplir de mejor manera sus roles familiares (abuelo/a, padre/madre, esposo/a, entre otros) después de la jubilación, los cuales eran difíciles de compatibilizar con las extensas horas dedicadas al trabajo previo. Sin embargo, algunos entrevistados también refieren haberse sentido completamente satisfechos antes de jubilar con su propio desempeño de roles de trabajador y compañero de trabajo.

\section{Percepciones negativas:}

- Extrañar:

“...por eso fue que después opté para llevarlos para allá conmigo hasta los campamentos, conseguí una casa, cierto, y me fui de poblador a Cullen y para estar con mi gente, porque yo los extrañaba" (E1,,63,L43).

- Insatisfacción:

"El rol de padre, bueno yo creo que el rol de padre no lo cumplí mucho entre comillas, no porque no lo haya querido hacer, sino que por la misma forma de trabajo que llevaba" (E3,H,63,L63).

Respecto de los cambios producidos en los roles, las personas refieren extrañar el desempeño de estos luego del proceso de la jubilación, situación que genera en ellos insatisfacción generada por el proceso de cambio. En relación a los conceptos antes mencionados, se analizó que el extrañar el desempeño de los roles, influye en el sentimiento de insatisfacción en los individuos. Los sujetos se sintieron insatisfechos con ciertos roles, ya que no lograban desempeñarlos de manera óptima. Es por ello, que paralelamente surge el sentimiento de extrañar, lo que antes hacían y hoy no se realiza, debido a que no contaban con el tiempo suficiente, específicamente para ejecutar los roles familiares debido al escaso tiempo con el cual disponían para desempeñarlos. Luego de jubilarse, las personas también refieren extrañar, pero en esta oportunidad en relación a los roles que mantenían cuando trabajaban (rol de compañero, trabajador, entre otros).

En definitiva, como lo grafica la figura $\mathrm{N}^{\circ} 1$ acerca del modelo explicativo-relacional sobre los cambios producidos en el proceso de la jubilación, es posible afirmar que a partir de los cambios que conlleva el proceso de la jubilación, en relación a los roles y rutinas de las personas mayores jubiladas, se observan percepciones positivas y/o negativas, respecto de las mismas, existiendo mayor cantidad de percepciones en relación a las rutinas, que a los roles desempeñados.

Por otro lado, se demuestra que si hay cambios en las rutinas de las personas, habrá una variación en el cumplimiento de roles, volviéndose algunos pasivos y activando otros, haciendo que las rutinas se vean modificadas en base al cumplimiento de estos.

Una vez que los entrevistados finalizan la etapa laboral, perciben este proceso como dificultoso, ya que comienzan a preocuparse por el gran tiempo libre que tendrán, tiempo 
con el cual no contaban cuando se encontraban trabajando activamente, debido a que las rutinas estaban estructuradas por las actividades laborales establecidas. Es por esto que las personas buscan involucrarse en nuevas actividades de participación social que son de interés personal; las cuales a su vez producen satisfacción en el individuo, haciendo posible el manejo en forma autónoma de su tiempo.

Paralelamente, surge la preocupación en el ámbito económico, debido que al jubilar se reduce considerablemente el ingreso monetario y es por esta situación que estas personas, comienzan la búsqueda de actividades que les proporcionen estos ingresos.

En relación a roles, los entrevistados manifiestan satisfacción con respecto al cumplimiento de roles significativos después del cese laboral, creando lazos afectivos que repercuten positivamente en la vida de la persona y que no fueron aprovechados en la etapa previa a la jubilación, permitiéndoles compartir más tiempo con personas que cumplen un papel fundamental en su diario vivir, o realizar planes que tenían pensados desde antes y que debido al poco tiempo que disponían no habían logrado realizar.

Dentro de los resultados obtenidos, se puede observar que los entrevistados descubren el sentido de la vida a través de las actividades que son significativas para ellos, donde a pesar de tener percepciones tanto positivas como negativas luego del proceso de jubilación, logran adaptarse al nuevo contexto en el cual se encuentran.

Como síntesis, se reafirma la vital importancia que la Terapia Ocupacional le asigna a la ocupación, elemento que puede promover el bienestar y del cual depende en gran medida la participación e inclusión social de los adultos mayores. 
Figura $\mathrm{N}^{\circ}$ 1: Modelo explicativo-relacional sobre los cambios producidos en el proceso de la jubilación.

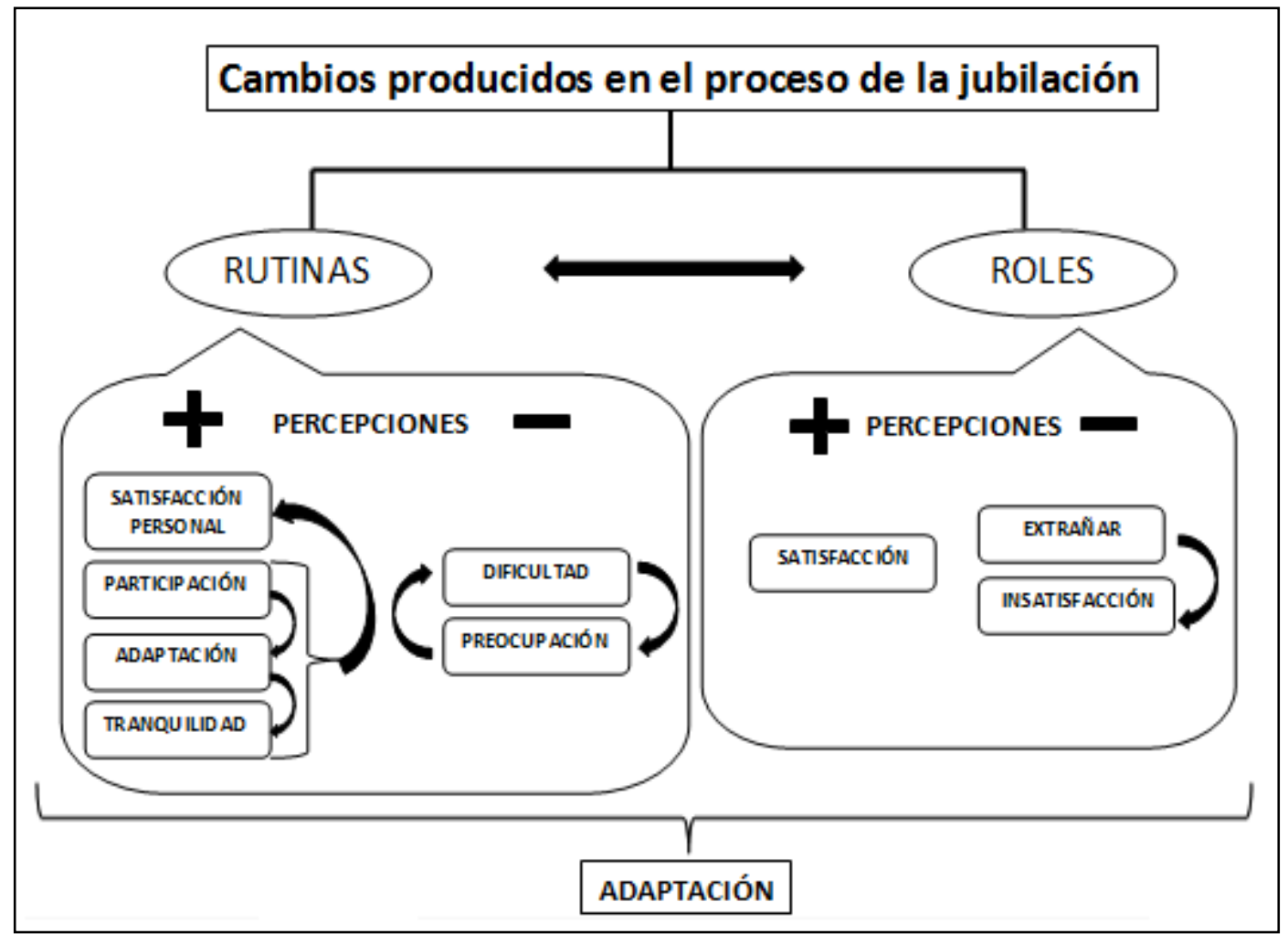

Fuente: Autoras

\section{DISCUSIÓN}

La investigación surge por la necesidad de profundizar sobre una temática nunca antes revisada en la región de Magallanes, desde la particular visión de la Ciencia de la Ocupación y la Terapia Ocupacional. A partir de ésta se puede concluir que:

- Se producen cambios en los patrones de ejecución de los adultos mayores luego de vivenciar el proceso de jubilación.

- Se producen cambios en la percepción de los adultos mayores, en relación a sus rutinas y roles, luego de vivenciar la jubilación.

- Los adultos mayores jubilados perciben mayores cambios asociados a las rutinas que en los roles que desempeñan.

- Los adultos mayores tienen percepciones positivas y negativas en relación a sus rutinas y roles post jubilación.

- Las percepciones de los adultos mayores están influenciadas por el tiempo del cual disponían antes versus el actual, lo que incide en la estructuración de sus rutinas. Hoy ellos estructuran independientemente su tiempo para realizar actividades significativas y de su propio interés, lo que les genera satisfacción personal, tranquilidad, participación social y una adaptación al proceso.

- A mayor participación en actividades significativas, mayor es la capacidad de adaptación de la persona al proceso de jubilación. 
- En los primeros meses surgen principalmente percepciones negativas en los individuos con respecto a los cambios producidos en sus rutinas y roles. Con el tiempo consiguen superarlo, logrando adaptarse al nuevo contexto al que se enfrentan, debido a que poseen importantes redes de apoyo en donde influye primordialmente su familia.

- Los entrevistados adquieren mayores actividades que son significativas para ellos, ya que disponen de mayor tiempo para organizarse y realizarlas.

La jubilación es un cambio importante para las personas debido a que modifica la estructura de las funciones que realiza y los obligan a asumir un nuevo rol. Velásquez (1999) menciona los tipos de jubilación existentes: jubilación voluntaria, forzosa, normal, anticipada y retrasada, destacándose principalmente dentro de los participantes de esta investigación, la jubilación normal y la anticipada. Por otra parte, Agulló-Tomás (2001) menciona que se presentan cinco tipos de actitudes respecto al proceso de la jubilación, el rechazo, aceptación, liberación, oportunidad y ambivalencia, incidiendo en los entrevistados fundamentalmente tres tipos, la aceptación, liberación y ambivalencia.

Es importante que los adultos mayores después del proceso de la jubilación logren organizar y utilizar su tiempo libre, ya que según Moragas, "el jubilado recibe el capital de su tiempo libre, pero si no sabe cómo invertirlo y mejorar su calidad de vida, se convierte en una carga, más que en una oportunidad" (Moragas, 1989).

Existen jubilados que a pesar de encontrarse en esta condición no permanecen retirados laboralmente, ya que continúan realizando actividades del cual obtienen ingresos monetarios. Se ha visto en la actualidad a más adultos mayores, a diferencia de las generaciones anteriores, que presentan capacidades para seguir activos y con interés en ampliar sus posibilidades de desarrollo personal y comunitario. Echenique (2006), describe al adulto mayor con "la capacidad de mantenerse en una actitud de vigencia, en oposición a la antigua denominación de anciano y vejez, cuya connotación se asociaba a incapacidad, invalidez y enfermedad".

Por otro lado, el dejar de trabajar genera disminución en el status social de las personas, ya que perciben que la sociedad los invalida, y si a eso se le suma la visión negativa que tiene el mismo adulto mayor respecto a la jubilación, directamente relacionado con la disminución de sus ingresos, la pérdida de relaciones interpersonales y el dejar de realizar la actividad que le demandaba mayor parte del tiempo, genera en ellos la auto-invalidación.

A partir de los antecedentes estudiados, y como sugerencias para la problemática presentada, se podrían implementar apoyos durante el proceso de jubilación y posterior a él, como por ejemplo:

- Desarrollar programas para adultos mayores de la región de Magallanes y Antártica Chilena, enfocados en la preparación previa para una jubilación satisfactoria, de manera que logren adaptarse a este proceso, organizando de mejor manera su tiempo. 
- Crear programas para el emprendimiento de proyectos, que estén al alcance de los adultos mayores de la región de Magallanes y Antártica Chilena.

- Realizar talleres de adaptación en relación a los cambios que se producen con la jubilación, para que los adultos mayores de la región de Magallanes y Antártica Chilena, se reorganicen y estructuren sus rutinas, posterior a la jubilación, de manera de favorecer su bienestar y su calidad de vida.

- Conseguir que los adultos mayores de la región de Magallanes y Antártica Chile, logren involucrarse en distintas actividades de su agrado, generando una mejor y más rápida adaptación a los cambios producidos por el proceso de jubilación.

Durante la realización del presente proceso investigativo, fue posible distinguir algunas limitaciones, las que podrían ser aprovechadas para la realización de futuras investigaciones en esta línea, en las cuales se podría profundizar con mayor énfasis.

Entre estos principales hallazgos se destacan:

- Se desconocen los mecanismos por los cuales las mujeres se adaptan con mayor facilidad a los cambios que se producen en el proceso de jubilación, en comparación a los hombres.

- También se desconoce el por qué para las mujeres es más fácil expresar sus percepciones con respecto a los cambios producidos en sus rutinas y roles, a diferencia de los hombres.

- Por otra parte es una limitación no contar con estadísticas regionales que indiquen información con respecto los factores socioeconómicos de adultos mayores jubilados.

\section{AGRADECIMIENTOS}

Nuestros más sinceros agradecimientos a aquellos Adultos Mayores que fueron partícipes de la investigación, así como también a quienes guiaron este trabajo, Terapeuta Ocupacional, Magíster en Educación Cristian Aranda Farías y Terapeuta Ocupacional, Magíster en Educación Andrea Yupanqui Concha. Y por último a cada una de nuestras familias. 


\section{REFERENCIAS BIBLIOGRAFICAS}

- Agulló-Tomás, M. S. (2001). Mayores, actividad y jubilación: Una aproximación psico-sociológica. Madrid, IMSERSO.

- Asociación Americana de Terapia Ocupacional (2010). Marco de trabajo para la práctica de Terapia Ocupacional: Dominio y Proceso. España, 2da Edición, Revista TOG.

- Christiansen, C. \& Baum, M. (eds.). (1997). Occupational Therapy: Enabling function and wellbing. Thorofare, NJ: Slack.

- Crepeau, Cohn, Schell, (2011). Willard \& Spackman. Terapia Ocupacional. Argentina: Panamericana.

- Echenique, L. y Arcos, M.E. (2006). Estudio de sexualidad en la tercera edad. Tesis de licenciatura publicada, Universidad Austral de Chile, Valdivia, Chile.

- Glaser, B. \& Strauss, A. (1967). The discovery of grounded grounded theory strategies for qualitative researsh. Chicago, Aldine Pub.

- Moragas, R. (1989). La jubilación. Un enfoque positivo. Grijalbo, Barcelona.

- Moreno, E. \& Miles, J. (2003). Adultos Mayores por Regiones, Comunas y Porcentajes (documento de trabajo). Instituto Nacional de Estadísticas (INE).

- Taylor, S. J., \& Bogdan, R. (1987). Introducción a los métodos cualitativos de investigación. Barcelona: Paidós Ibérica, S.A.

- Velásquez, M. (1999). La formación de formadores para los programas universitarios de mayores. EA, Escuela abierta: Revista de Investigación Educativa, 3, pp. 133-162. Recuperado el 12 de diciembre de 2011, de http://dialnet.unirioja.es/servlet/listaarticulos?tipo_busqueda=ANUALIDAD\&revist a busqueda $=511 \&$ clave busqueda $=1999$ 